\title{
Non-Markovianity Hierarchy of Gaussian Processes and Quantum Amplification
}

\author{
Pietro Liuzzo-Scorpo, ${ }^{1}$ Wojciech Roga, ${ }^{2}$ Leonardo A. M. Souza, ${ }^{3,1}$ Nadja K. Bernardes, ${ }^{4}$ and Gerardo Adesso ${ }^{1}$ \\ ${ }^{1}$ Centre for the Mathematics and Theoretical Physics of Quantum Non-Equilibrium Systems, School of Mathematical Sciences, \\ The University of Nottingham, University Park, Nottingham NG7 2RD, United Kingdom \\ ${ }^{2}$ Department of Physics, University of Strathclyde, John Anderson Building, 107 Rottenrow, Glasgow G4 ONG, United Kingdom \\ ${ }^{3}$ Universidade Federal de Viçosa-Campus Florestal, LMG818 Km6, Minas Gerais, Florestal 35690-000, Brazil \\ ${ }^{4}$ Departamento de Física, Universidade Federal de Minas Gerais, Belo Horizonte, Caixa Postal 702, 30161-970, Brazil
}

(Received 1 November 2016; published 31 January 2017)

\begin{abstract}
We investigate the dynamics of Gaussian states of continuous variable systems under Gaussianitypreserving channels. We introduce a hierarchy of such evolutions encompassing Markovian and weakly and strongly non-Markovian processes and provide simple criteria to distinguish between the classes, based on the degree of positivity of intermediate Gaussian maps. We present an intuitive classification of all one-mode Gaussian channels according to their non-Markovianity degree and show that weak non-Markovianity has an operational significance, as it leads to a temporary phase-insensitive amplification of Gaussian inputs beyond the fundamental quantum limit. Explicit examples and applications are discussed.
\end{abstract}

DOI: 10.1103/PhysRevLett.118.050401

Introduction.-Non-Markovian evolutions of open quantum systems have been extensively studied in recent years [1-3]. During these evolutions, memory effects appear in many forms $[1,2,4-16]$. These effects can lead to enhancements in quantum computation, e.g., for error correction or decoherence suppression [17-24], in quantum cryptography [25], limiting the information accessible to the eavesdropper, and possibly in the efficiency of certain processes at the intersection between quantum physics and biology [26-28]. Experimental techniques are now mature to investigate open quantum systems beyond the Markovian regime [29-39].

A quantum process defined by a completely positive (CP) dynamical map $\Lambda_{t}$ is Markovian if it is CP-divisible, i.e., such that an intermediate map $\tilde{\Lambda}_{t+\tau, t}$, defined by $\Lambda_{t+\tau}=\tilde{\Lambda}_{t+\tau, t} \Lambda_{t}$, is CP for all $t, \tau>0$. A CP map can indeed be represented by an interaction of the evolving system with an uncorrelated environment [40]: The lack of correlations at each step denotes a lack of memory and, hence, Markovianity. On the other hand, we recognize a nonMarkovian process when its description cannot be found among CP-divisible maps. In this case, correlations between the system and environment are essential at some stage.

The association of Markovian processes with CPdivisible maps results in important restrictions. For instance, the entanglement, mutual information, or quantum channel capacity cannot increase if a CP map is applied locally to the subsystems. Similarly, measures of state distinguishability, like fidelity or trace distance, are contractive under CP maps. A violation of CP-divisibility is then witnessed by the temporary increase of these quantities [5-13]. Proper measures of non-Markovianity rely on the direct examination of complete positivity of all intermediate maps [6,14-16]. A unified picture of several quantifiers of non-Markovianity has been presented in Ref. [15], where a hierarchy of non-Markovianity degrees was introduced, based on the smallest degree of positivity of intermediate maps.

Further important insight into non-Markovian processes is achieved considering evolutions of quantum states living in infinite-dimensional Hilbert spaces, such as states of light. These states are described by continuous variables $(\mathrm{CVs})$ related to quadratures (position and momentum operators) [41]. In the CV formalism, the complete positivity of maps amounts to the fulfilment of the uncertainty principle for any legitimate input state. Clearly, the monotonicity of distinguishability or entanglement under CP-divisible maps extends to CV systems as well.

In this Letter, we consider evolutions of Gaussian quantum states governed by Gaussianity-preserving processes (Gaussian maps). Taking inspiration from the line drawn in Ref. [15] for finite-dimensional processes and building on the methods of Ref. [14], where an elegant characterization of (non-) Markovianity was accomplished for Gaussian maps in terms of CP-divisibility, we identify a simple general hierarchy based on the divisibility degree of Gaussian maps with Gaussian inputs. This hierarchy, obtained by introducing an intermediate notion of Gaussian $k$-positivity and providing necessary and sufficient criteria for it, allows us to distinguish three classes of processes: Markovian and weakly and strongly non-Markovian.

We then classify all one-mode Gaussian channels according to their non-Markovianity degree, using an intuitive pictorial diagram divided in three regions, one per each class of the hierarchy. We relate the latter to possibilities and limitations for quantum amplification. In particular, weakly non-Markovian phase-insensitive channels allow, during some intermediate time, for the 
amplification of Gaussian inputs with less added noise than the fundamental quantum limit [42-45]. This provides a fascinating operational interpretation for weak nonMarkovianity, which had gone unnoticed before [15].

Gaussian states and Gaussian maps. - Given an $n$-mode CV system, Gaussian states $\rho$ are defined as those having a Gaussian characteristic function in phase space [41,46,47]. These states are fully characterized by the first and second statistical moments of their quadrature vector $\hat{O}=\left\{\hat{q}_{1}, \hat{p}_{1}, \ldots, \hat{q}_{n}, \hat{p}_{n}\right\}$, where $\hat{q}_{j}$ and $\hat{p}_{k}$ are canonically conjugate coordinates satisfying $\left[\hat{q}_{j}, \hat{p}_{k}\right]=(i / 2) \delta_{j k}$ (in natural units, $\hbar=1)$. The first moment vector $D=\langle\hat{O}\rangle$ is also called the displacement vector, while the second moments $\left(\sigma_{n}\right)_{j k}=\frac{1}{2}\left\langle\left\{\hat{O}_{j}, \hat{O}_{k}\right\}_{+}\right\rangle$form the covariance matrix $\sigma_{n}$. All physical states must satisfy the RobertsonSchrödinger uncertainty relation $\sigma_{n} \geq(i / 2) \Omega_{n}$, where

$$
\Omega_{n}=\left(\begin{array}{cc}
0 & 1 \\
-1 & 0
\end{array}\right)^{\oplus n}
$$

is the $n$-mode symplectic matrix [48].

Quantum channels that preserve Gaussianity of their inputs are known as Gaussian maps. A Gaussian map acting on $n$-mode Gaussian states is represented by a pair of $2 n \times 2 n$ matrices $(X, Y)$, with $Y$ symmetric, acting on the displacement vector $D$ and the covariance matrix $\sigma_{n}$ as follows [14,49-53]:

$$
D \rightarrow D^{\prime}=X D, \quad \sigma_{n} \rightarrow \sigma_{n}^{\prime}=X \sigma_{n} X^{T}+Y .
$$

A Gaussian map described by the pair $(X, Y)$ is $\mathrm{CP}$ if and only if the following well-known inequality is fulfilled [14,52-54]:

$$
Y-\frac{i}{2} \Omega_{n}+\frac{i}{2} X \Omega_{n} X^{T} \geq 0 .
$$

This inequality can be obtained from the Stinespring dilation theorem [40], i.e., considering the Gaussian map as the result of a Gaussian unitary evolution acting on the system and environment, initialized in an uncorrelated $(n+m)$-mode Gaussian state, followed by a partial trace over the $m$ environment modes, $\sigma_{n} \rightarrow \sigma_{n}^{\prime}=\operatorname{Tr}_{E}\left[S\left(\sigma_{n} \oplus \sigma_{m}^{E}\right) S^{T}\right]$; here one uses the fact that a Gaussian unitary is represented by a symplectic transformation $S \in \operatorname{Sp}(2 n+2 m, \mathbb{R})$ (i.e., one that preserves the symplectic matrix $\Omega$ ) acting by congruence on covariance matrices [41].

$k$-positivity of Gaussian maps.-We now introduce a notion of $k$-positivity for Gaussian maps with Gaussian inputs, inspired by the hierarchy of $k$-positivity for finitedimensional channels arising from Choi's theorem [55]. We define a Gaussian map acting on $n$-mode Gaussian inputs as $k$-positive $(k \mathrm{P})$ if its extension on $k$ additional modes is positive, i.e., if, for all $(n+k)$-mode Gaussian states described by covariance matrices $\sigma_{n+k} \geq(i / 2) \Omega_{n+k}$, it holds that

$$
\left(X \oplus \mathbb{1}_{k}\right) \sigma_{n+k}\left(X \oplus \mathbb{1}_{k}\right)^{T}+Y \oplus \mathbb{O}_{k} \geq \frac{i}{2} \Omega_{n+k} .
$$

Interestingly, we prove in the Appendix [56] that a Gaussian map with Gaussian inputs is $\mathrm{CP}$ if and only if it is $k \mathrm{P}$ with any $k \geq 1$. Precisely, we establish the following result.

Theorem 1. For any n, the CP condition (2) is equivalent to the $k \mathrm{P}$ condition (3) with $k=1$.

This means that, in the Gaussian scenario (unlike the general finite-dimensional case [57]), one has a very simple hierarchy of $k$-positivity, consisting of only three classes: completely positive ( $\mathrm{CP}, k=1)$, positive $(\mathrm{P}, k=0)$, and not positive (NP) Gaussian maps. We can derive a simple (and, to our knowledge, original) condition to distinguish between the latter two classes, in terms of the pair $(X, Y)$. Noting that for (3) to hold it suffices to check its validity on pure Gaussian states, whose covariance matrix can always be written as $\sigma=\frac{1}{2} S S^{T}$ with $S$ a symplectic transformation, we find that a Gaussian map with Gaussian inputs is positive $(k=0)$ if and only if

$$
\frac{1}{2} X S S^{T} X^{T}+Y-\frac{i}{2} \Omega_{n} \geq 0, \quad \forall S \in \operatorname{Sp}(2 n, \mathbb{R}) .
$$

Conditions (2) and (4) allow one to fully classify the positivity properties of any $(n \rightarrow n)$-mode Gaussian map described by the pair $(X, Y)$ acting on Gaussian inputs. The conditions can be easily generalized to $(n \rightarrow m)$-mode Gaussian maps.

Hierarchy of Gaussian non-Markovianity.-Gaussian processes which are continuous in time are represented by a pair of time-dependent matrices $\left(X_{t}, Y_{t}\right)$ acting as in (1). Since we are interested in the divisibility properties of these maps, we can follow the approach of Ref. [14] and study the positivity of the intermediate map $\left(X_{\tau}(t), Y_{\tau}(t)\right)$ acting on the evolving system between times $t$ and $t+\tau$ and affecting the covariance matrix as usual, $\sigma(t) \rightarrow \sigma(t+\tau)=$ $X_{\tau}(t) \sigma(t) X_{\tau}^{T}(t)+Y_{\tau}(t)$, with [14]

$X_{\tau}(t)=X_{t+\tau} X_{t}^{-1}, \quad Y_{\tau}(t)=Y_{t+\tau}-X_{\tau}(t) Y_{t} X_{\tau}^{T}(t)$.

Our aim is now to provide a complete (non-) Markovianity hierarchy of Gaussian maps. We first recall that, imposing complete positivity of the intermediate map for all $t, \tau>0$, one obtains the condition for a Markovian evolution as in [14]:

$$
\frac{i}{2} X_{\tau}(t) \Omega X_{\tau}^{T}(t)+Y_{\tau}(t)-\frac{i}{2} \Omega \geq 0 .
$$

Any Gaussian map not complying with condition (6) at some intermediate times is non-Markovian [14]. We can now add an extra layer to such a dichotomic characterization. Namely, if not all intermediate maps are CP, i.e., if for some times condition (6) is violated, but the positivity condition

$\frac{1}{2} X_{\tau}(t) S S^{T} X_{\tau}^{T}(t)+Y_{\tau}(t)-\frac{i}{2} \Omega \geq 0, \quad \forall S \in \operatorname{Sp}(2 n, \mathbb{R})$

holds for all the maps, then the evolution is said to be weakly non-Markovian. Finally, if there is at least one intermediate map violating (7), the process is strongly non-Markovian. 
It is worth noting that, to check $\mathrm{CP}$ (respectively, $\mathrm{P}$ ) divisibility of the map $\left(X_{t}, Y_{t}\right)$, it suffices to verify that inequality (6) [respectively, (7)] holds in the limit of small $\tau$, since the composition of an arbitrary number of intermediate $\mathrm{CP}(\mathrm{P})$ maps is $\mathrm{CP}(\mathrm{P})$.

Complete classification of one-mode Gaussian maps.In what follows, we focus on one-mode quantum Gaussian processes. From the global map $\left(X_{t}, Y_{t}\right)$, we can construct, thanks to (5), the intermediate maps given by the pairs of $2 \times 2$ matrices $\left(X_{\tau}(t), Y_{\tau}(t)\right)$. In the limit of small $\tau, X_{\tau}(t)$ and $Y_{\tau}(t)$ are close to the identity and to the null matrix, respectively. Expanding these matrices up to first order in $\tau$, we get

$$
\begin{aligned}
X_{\tau}(t) & =\left(1+\epsilon_{t} \tau\right) \mathbb{1}+\tau \mathcal{X}(t)+o\left(\tau^{2}\right), \\
Y_{\tau}(t) & =\tau \mathcal{Y}(t)+o\left(\tau^{2}\right),
\end{aligned}
$$

where $\mathcal{X}(t)$ and $\mathcal{Y}(t)$ are arbitrary real matrices, with $\mathcal{Y}(t)$ being symmetric. The following two theorems (proofs in the Appendix [56]) then completely characterize the degree of Gaussian (non-) Markovianity of any one-mode Gaussian map given by $\left(X_{t}, Y_{t}\right)$, in terms of the three real parameters

$$
\begin{gathered}
\epsilon_{t} \equiv \frac{d}{d t} \ln \left(\sqrt{\left|\operatorname{det} X_{t}\right|}\right), \\
\delta_{t} \equiv\left(\operatorname{det} X_{t}\right)^{2} \operatorname{det}\left(\frac{d}{d t}\left(X_{t}^{-1} Y_{t} X_{t}^{-T}\right)\right), \\
\kappa_{t} \equiv \frac{d}{d t} \operatorname{tr} Y_{t}-2 \operatorname{tr}\left(Y_{t} \frac{d}{d t} \ln \left|X_{t}\right|\right) .
\end{gathered}
$$

Theorem 2. A one-mode Gaussian process given by $\left(X_{t}, Y_{t}\right)$ is CP-divisible if, for all $t>0$, it holds that $\delta_{t} \geq \epsilon_{t}^{2}$ and $\kappa_{t} \geq 0$.

Theorem 3. A one-mode Gaussian process given by $\left(X_{t}, Y_{t}\right)$ is divisible into positive intermediate maps (P-divisible) if, for all $t>0$, it holds that $\delta_{t} \geq \frac{1}{4}\left(\left|\epsilon_{t}\right|-\epsilon_{t}\right)^{2}$ and $\kappa_{t} \geq 0$.

The Gaussian processes for which Theorem 2 is satisfied are Markovian. Those for which Theorem 3 is satisfied while Theorem 2 is not are weakly non-Markovian. Those for which Theorem 3 is not satisfied are strongly non-Markovian.

Let us now define

$$
\mu_{t} \equiv \begin{cases}\operatorname{sgn}\left(\kappa_{t}\right) \sqrt{\delta_{t}}, & \text { for } \delta_{t} \geq 0, \\ -\sqrt{\left|\delta_{t}\right|}, & \text { for } \delta_{t}<0 .\end{cases}
$$

Because of Theorems 2 and 3, for a one-mode Gaussian process we can then distinguish three regions in the space of parameters $\epsilon$ and $\mu$ as shown in Fig. 1, which correspond to the intermediate map being, respectively, CP, P, and NP:

$$
\begin{aligned}
& \Upsilon_{\mathrm{CP}} \equiv\{(\epsilon, \mu)|\mu \geq| \epsilon \mid\}, \\
& \Upsilon_{\mathrm{P}} \equiv\{(\epsilon, \mu)|2 \mu \geq| \epsilon \mid-\epsilon\}, \\
& \Upsilon_{\mathrm{NP}} \equiv \mathbb{R}^{2} \backslash \Upsilon_{\mathrm{P}} .
\end{aligned}
$$

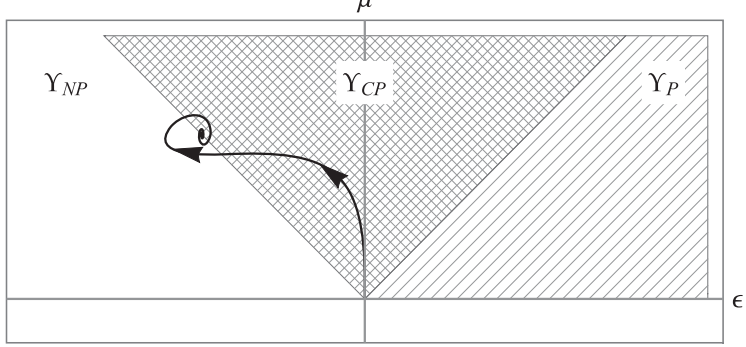

FIG. 1. Pictorial diagram of parameters $(\epsilon, \mu)$ characterizing one-mode Gaussian intermediate maps. The diagonal striped pattern corresponds to the P- but not CP-divisible region $\Upsilon_{\mathrm{P} \backslash \mathrm{CP}}$. The crosshatch pattern identifies the CP-divisible region $\Upsilon_{\mathrm{CP}}$. The white region corresponds to $\Upsilon_{\mathrm{NP}}$. A path on the diagram denotes a process with parameters changed continuously in time. The solid black path represents a quantum Brownian motion process as described in the text.

A similar diagram can be found, e.g., in Refs. [54,58,59]. However, the parameters there characterize global quantum channels, so regions analogous to $\Upsilon_{\mathrm{P} \backslash \mathrm{CP}} \equiv \Upsilon_{\mathrm{P}} \backslash \Upsilon_{\mathrm{CP}}$ and $\Upsilon_{\mathrm{NP}}$ are denoted as nonphysical. Here, since the diagram is built for intermediate maps of a globally $\mathrm{CP}$ process, which by themselves do not need to be $\mathrm{CP}$, these regions are permitted.

We can in fact fully classify the Gaussian (non-) Markovianity degree of any one-mode Gaussian process by studying the paths $\Gamma_{t} \equiv\left\{\left(\epsilon_{s}, \mu_{s}\right)\right\}_{s=0}^{t}$ defined by its intermediate maps on the $(\epsilon, \mu)$ diagram of Fig. 1. If an evolution is Markovian, then the trajectory will be confined at all times in the $\Upsilon_{\mathrm{CP}}$ region, $\Gamma_{t} \in \Upsilon_{\mathrm{CP}} \forall t>0$. If at some times the trajectory trespasses in the $\Upsilon_{\mathrm{P} \backslash \mathrm{CP}}$ region but never trespasses in the $\Upsilon_{\mathrm{NP}}$ one, i.e., if $\Gamma_{t} \in \Upsilon_{\mathrm{P}} \forall t>0$ and $\exists s:\left(\epsilon_{s}, \mu_{s}\right) \notin \Upsilon_{\mathrm{CP}}$, then the evolution is weakly nonMarkovian. If at some times the trajectory crosses into the $\Upsilon_{\mathrm{NP}}$ region, i.e., $\exists s:\left(\epsilon_{s}, \mu_{s}\right) \notin \Upsilon_{\mathrm{P}}$, then the evolution is strongly non-Markovian.

Phase-insensitive maps: Allowed trajectories and examples.-We now analyze in more detail the physical constraints imposed on processes described by CP maps $\left(X_{t}, Y_{t}\right)$, transforming Gaussian states from an initial $t=0$ to a later time $t$. For ease of illustration, we will focus on the special case of phase-insensitive maps, which encompass the most physically relevant bosonic processes, such as quantum Brownian motion and amplitude damping $[12,14,25,37,60-62]$. These have intermediate maps of the form $X_{\tau}(t)=\left(1+\epsilon_{t} \tau\right) \mathbb{1}, \quad Y_{\tau}(t)=\mu_{t} \tau \mathbb{1}$, with $\mu_{t} \equiv$ $\operatorname{sgn}\left(\kappa_{t}\right) \sqrt{\delta_{t}}$, obtained by setting $\mathcal{X}(t)=\mathbb{O}$ and $\mathcal{Y}(t)=$ $\mu_{t} \mathbb{1}$ in (8). Applying the composition law for Gaussian maps, it is easy to show that the global map from $t=0$ to $t=N \tau$, such that $\lim _{N \rightarrow \infty} \lim _{\tau \rightarrow 0} N \tau=t>0$, is

$X_{t}=e^{\int_{0}^{t} \epsilon_{s} d s} \mathbb{1}, \quad Y_{t}=\left(e^{2 \int_{0}^{t} \epsilon_{s} d s} \int_{0}^{t} \mu_{r} e^{-2 \int_{0}^{r} \epsilon_{s} d s} d r\right) \mathbb{1}$.

A paradigmatic and widely studied example (see, e.g., $[60,61]$, and references therein) is quantum Brownian 
motion. With a secular and weak-coupling approximation, the master equation is given by $\dot{\rho}_{t}=\left(\Delta_{t}+\gamma_{t}\right) / 2\left[a \rho_{t} a^{\dagger}-\right.$ $\left.\left\{a^{\dagger} a, \rho_{t}\right\}_{+}\right]+\left(\Delta_{t}-\gamma_{t}\right) / 2\left[a^{\dagger} \rho_{t} a-\left\{a a^{\dagger}, \rho_{t}\right\}_{+}\right]$, where $\hat{a}, \hat{a}^{\dagger}$ are the ladder operators satisfying $\left[\hat{a}, \hat{a}^{\dagger}\right]=1$, while $\Delta_{t}$ and $\gamma_{t}$ are, respectively, the diffusion and damping coefficients, which depend on the spectral density of the bath. The evolved covariance matrix of a one-mode Gaussian state undergoing this dynamics is $\sigma(t)=$ $\left(e^{-\int_{0}^{t} \gamma_{s} d s} \mathbb{1}\right) \sigma(0)\left(e^{-\int_{0}^{t} \gamma_{s} d s} \mathbb{1}\right)+e^{-2 \int_{0}^{t} \gamma_{s} d s} \int_{0}^{t} e^{2 \int_{0}^{s} \gamma_{r} d r} \Delta_{s} d s \mathbb{1}$, which corresponds to the map $\left(X_{t}, Y_{t}\right)$ given by (14) with the substitutions $\epsilon_{t} \rightarrow-\gamma_{t}$ and $\mu_{t} \rightarrow \Delta_{t}$. A trajectory on the $(\epsilon, \mu)$ plane, for a system with characteristic frequency $\omega_{0}$ and a zero-temperature bath with Ohmic spectral density $J(\omega)=\omega e^{-\omega / \omega_{c}}$ and cutoff frequency $\omega_{c}=\omega_{0} / 2$, is depicted in Fig. 1.

More generally, to have a physical evolution from a composition of infinitesimal phase-insensitive maps, we must impose the CP condition (2) on the global map $\left(X_{t}, Y_{t}\right)$ given by (14). The eigenvalues of the lhs of (2) are in this case $\Lambda_{ \pm}= \pm \frac{1}{2}+e^{2 \int_{0}^{t} \epsilon_{s} d s}\left(\mp \frac{1}{2}+\int_{0}^{t} e^{-2 \int_{0}^{r} \epsilon_{s} d s} \mu_{r} d r\right)$. The conditions $\Lambda_{ \pm} \geq 0$ can be rewritten as (see the Appendix [56])

$$
\int_{0}^{t} e^{-2 \int_{0}^{s} \epsilon_{s} d s}\left(\mu_{r} \pm \epsilon_{r}\right) d r \geq 0, \quad \forall t>0 .
$$

As expected, these conditions are weaker than the condition for CP-divisibility, allowing the trajectories in the diagram of Fig. 1 to go beyond the region $\Upsilon_{\mathrm{CP}}$. However, the following constraint on the physical paths can be derived. By expanding the lhs of inequalities (15) at first order in $t$, we get $\mu_{0} \geq\left|\epsilon_{0}\right|$; that is, the trajectory must begin in the CP region $\Upsilon_{\mathrm{CP}}$. Moreover, if it starts on the boundaries of $\Upsilon_{\mathrm{CP}}$, i.e., $\mu_{0}=\left|\epsilon_{0}\right|$, then $\dot{\mu}_{0} \geq\left|\dot{\epsilon}_{0}\right|$. This tells us not only that the trajectory must start in the CP-divisibility region, but it has to have an initial "speed" such that it will remain in there for the immediate subsequent time. A path which starts in the origin, then moves along the boundary of the crosshatched region up to a time $t_{i}$, and then trespasses in either region $\Upsilon_{\mathrm{NP}}$ or $\Upsilon_{\mathrm{P} \backslash \mathrm{CP}}$ is not allowed.

Operational significance of Gaussian non-Markovianity degrees.-The significance of the last no-go rule is related to fundamental physical properties. Suppose indeed that at time $t=0$ an initial state is described by a thermal covariance matrix $\sigma=\operatorname{diag}\{\nu, \nu\}$, with $\nu \geq \frac{1}{2}$. Under the action of the map (14) at time $\tilde{t}>0$, the product of the canonical variances is $\left\langle\hat{q}^{2}\right\rangle\left\langle\hat{p}^{2}\right\rangle=e^{4 \int_{0}^{\tilde{t}} \epsilon_{s} d s}\left(\nu+\int_{0}^{\tilde{t}} \mu_{r} e^{-2 \int_{0}^{r} \epsilon_{s} d s} d r\right)^{2}$. If $\Lambda_{-}<0$, we obtain $\left\langle\hat{q}^{2}\right\rangle\left\langle\hat{p}^{2}\right\rangle<e^{2 \int_{0}^{t} \epsilon_{s} d s}\left[e \int_{0}^{\tilde{t}} \epsilon_{s} d s \nu-\sinh \left(\int_{0}^{\tilde{t}} \epsilon_{s} d s\right)\right]^{2}$, which for a pure initial state (i.e., the vacuum or a Glauber coherent state, with $\nu=\frac{1}{2}$ ) reduces to $\left\langle\hat{q}^{2}\right\rangle\left\langle\hat{p}^{2}\right\rangle<\frac{1}{4}$, i.e., to a violation of the uncertainty principle, which is not physically admitted. Indeed, a trajectory lying along the border between $\Upsilon_{\mathrm{CP}}$ and $\Upsilon_{\mathrm{NP}}$ (representing, e.g., a damping master equation with a generally time-dependent damping constant) preserves the purity of such a state. To better understand this, let us consider the limiting case of having a map such that $-\epsilon_{t}=\mu_{t}>0$ for $0<t<t_{i}$ and $-\epsilon_{t}>\mu_{t}$ for $t_{i}<t<\tilde{t}$. Up to $t_{i}$, the $X_{t}$ part of the map decreases both variances of the pure input state, while the noise added by the $Y_{t}$ part compensates the loss and the state remains pure. Then, for $t>t_{i}$, the noise introduced by $Y_{t}$ is not enough and the uncertainty relation is violated.

However, crossing the border during the evolution would be possible if the preceding dynamics shrank the state domain of the intermediate map such that its subsequent action, corresponding to a temporary dilation of this domain, would not violate the uncertainty relation. The non-Markovian effect, manifested in the dilation of the volume of the physical states accessible during the dynamics, can then be seen as a backflow of information from the environment into the system [9].

Let us now comment on the other border of the CP region, between $\Upsilon_{\mathrm{CP}}$ and $\Upsilon_{\mathrm{P} \backslash \mathrm{CP}}$. For any dynamics with added noise $\left(\mu_{t}>0\right)$, a trajectory along this border is such that $\epsilon_{t}=\mu_{t}>0$, which is responsible for an amplification, that is, the multiplication of the displacement vector $D$ by a factor greater than 1 and a corresponding increase of the variances. Along such a path, the noise added is the minimum allowed for quantum linear amplifiers [44]. Crossing this border into the $\Upsilon_{\mathrm{P} \backslash \mathrm{CP}}$ region at a time $t_{i}>0$ is allowed only if the noise added up to that time is sufficient to permit a subsequent amplification beyond the quantum limit. This is possible thanks to correlations established between the system and environment during the preceding evolution. We can conclude therefore that a Gaussian phase-insensitive process (with added noise) is weakly non-Markovian if at any moment in time one observes that, although the covariances increase, a Gaussian state evolving under such a process is amplified, beating the quantum limit. This provides an operational interpretation for the elusive phenomenon of weak nonMarkovianity in the context of quantum amplification.

Conclusions.-This Letter introduced a meaningful hierarchy of non-Markovianity for CV Gaussian processes and established its physical significance. We provided a necessary and sufficient condition for positivity of a Gaussian map acting on Gaussian inputs. Applying this to intermediate maps, we then distinguished three main types of Gaussian processes: Markovian and weakly and strongly non-Markovian ones.

In the one-mode case, we gave a simple prescription to identify to which class a Gaussian map belongs, based on its representation as a path $\left(\epsilon_{t}, \mu_{t}\right)$ in a two-dimensional diagram, where $\epsilon_{t}$ and $\mu_{t}$ can be computed explicitly from the pair of matrices $\left(X_{t}, Y_{t}\right)$ describing the action of the map. We also studied, in the physically relevant case of phase-insensitive channels, the constraints on these paths due to the requirement of having a global CP map. This allowed us to give a physical interpretation to weakly and strongly non-Markovian processes in terms of amplification beyond the quantum limit and of information backflow from the environment, respectively. 
These findings can be of importance for quantum cryptography [25]. An eavesdropper with access to knowledge whether a given communication channel is weakly or strongly non-Markovian can amplify a state in such a way that the legitimate parties may find it too noisy to be useful, discarding it. Moreover, if the legitimate parties do not fully control the way the shared state is prepared, unexpected behavior can be observed if possible non-Markovian effects are ignored.

We finally note that in all the Gaussian processes we considered explicitly (e.g., the quantum Brownian model and the damping model), we found either instances of Markovian or strongly non-Markovian evolutions but no weakly non-Markovian ones. This may be due to the fact that all these processes admit a final state at thermal equilibrium with the environment. Some purely weak non-Markovian processes might be retrieved in the case where an evolution in an active environment that pumps energy into the system is analyzed. Investigating memory effects in such processes deserves further investigation.

We thank Sabrina Maniscalco, Dariusz Chruściński, John Jeffers, Marco Piani, Gianpaolo Torre, and Fabrizio Illuminati for discussions. This work was supported by the United Kingdom EPSRC Quantum Imaging Hub (Grant No. EP/M01326X/1), the European Research Council (ERC) Starting Grant GQCOP (Grant No. 637352), the Foundational Questions Institute Physics of the Observer Programme (Grant No. FQXi-RFP-1601), the Brazilian Agencies CAPES (Grant No. 6842/2014-03) and CNPq (Grant No. 470131/2013-6), and the University of Nottingham (Graduate School Travel Prize 2015).

[1] Á. Rivas, S. F. Huelga, and M. B. Plenio, Rep. Prog. Phys. 77, 094001 (2014).

[2] H.-P. Breuer, E.-M. Laine, J. Piilo, and B. Vacchini, Rev. Mod. Phys. 88, 021002 (2016).

[3] I. de Vega and D. Alonso, Rev. Mod. Phys. 89, 15001 (2017).

[4] M. M. Wolf, J. Eisert, T. S. Cubitt, and J. I. Cirac, Phys. Rev. Lett. 101, 150402 (2008).

[5] H.-P. Breuer, E.-M. Laine, and J. Piilo, Phys. Rev. Lett. 103, 210401 (2009).

[6] Á. Rivas, S. F. Huelga, and M. B. Plenio, Phys. Rev. Lett. 105, 050403 (2010).

[7] X.-M. Lu, X. Wang, and C. P. Sun, Phys. Rev. A 82, 042103 (2010)

[8] E.-M. Laine, J. Piilo, and H.-P. Breuer, Phys. Rev. A 81, 062115 (2010).

[9] S. Lorenzo, F. Plastina, and M. Paternostro, Phys. Rev. A 88, 020102 (2013).

[10] B. Bylicka, D. Chruściński, and S. Maniscalco, Sci. Rep. 4, 5720 (2014).

[11] H. S. Dhar, M. N. Bera, and G. Adesso, Phys. Rev. A 91, 032115 (2015).

[12] L. A. M. Souza, H. S. Dhar, M. N. Bera, P. Liuzzo-Scorpo, and G. Adesso, Phys. Rev. A 92, 052122 (2015).

[13] F. Buscemi and N. Datta, Phys. Rev. A 93, 012101 (2016).
[14] G. Torre, W. Roga, and F. Illuminati, Phys. Rev. Lett. 115, 070401 (2015).

[15] D. Chruścinski and S. Maniscalco, Phys. Rev. Lett. 112, 120404 (2014).

[16] F. A. Pollock, C. Rodrguez-Rosario, T. Frauenheim, M. Paternostro, and K. Modi, arXiv:1512.00589.

[17] E.-M. Laine, H.-P. Breuer, and J. Piilo, Sci. Rep. 4, 4620 (2014).

[18] G. He, J. Zhang, J. Zhu, and G. Zeng, Phys. Rev. A 84, 034305 (2011).

[19] M. J. Biercuk, H. Uys, A. P. VanDevender, N. Shiga, W. M. Itano, and J. J. Bollinger, Nature (London) 458, 996 (2009).

[20] C. J. Myatt, B. E. King, Q. A. Turchette, C. A. Sackett, D. Kielpinski, W. M. Itano, C. Monroe, and D. J. Wineland, Nature (London) 403, 269 (2000).

[21] F. Verstraete, M. M. Wolf, and J. I. Cirac, Nat. Phys. 5, 633 (2009).

[22] Q.-J. Tong, J.-H. An, H.-G. Luo, and C. H. Oh, Phys. Rev. A 81, 052330 (2010).

[23] Y.-Q. Lü, J.-H. An, X.-M. Chen, H.-G. Luo, and C. H. Oh, Phys. Rev. A 88, 012129 (2013).

[24] S. Haseli, G. Karpat, S. Salimi, A. S. Khorashad, F. F. Fanchini, B. Çakmak, G. H. Aguilar, S. P. Walborn, and P. H. Souto Ribeiro, Phys. Rev. A 90, 052118 (2014).

[25] R. Vasile, S. Maniscalco, M. G. A. Paris, H.-P. Breuer, and J. Piilo, Phys. Rev. A 84, 052118 (2011).

[26] M. Thorwart, J. Eckel, J. Reina, P. Nalbach, and S. Weiss, Chem. Phys. Lett. 478, 234 (2009).

[27] A. W. Chin, J. Prior, R. Rosenbach, F. Caycedo-Soler, S. F. Huelga, and M. B. Plenio, Nat. Phys. 9, 113 (2013).

[28] S. Huelga and M. Plenio, Contemp. Phys. 54, 181 (2013).

[29] B.-H. Liu, L. Li, Y.-F. Huang, C.-F. Li, G.-C. Guo, E.-M. Laine, H.-P. Breuer, and J. Piilo, Nat. Phys. 7, 931 (2011).

[30] A. Chiuri, C. Greganti, L. Mazzola, M. Paternostro, and P. Mataloni, Sci. Rep. 2, 968 (2012).

[31] J.-S. Tang, C.-F. Li, Y.-L. Li, X.-B. Zou, G.-C. Guo, H.-P. Breuer, E.-M. Laine, and J. Piilo, Europhys. Lett. 97, 10002 (2012).

[32] J.-S. Xu, K. Sun, C.-F. Li, X.-Y. Xu, G.-C. Guo, E. Andersson, R. Lo Franco, and G. Compagno, Nat. Commun. 4, 2851 (2013).

[33] B.-H. Liu, D.-Y. Cao, Y.-F. Huang, C.-F. Li, G.-C. Guo, E.-M. Laine, H.-P. Breuer, and J. Piilo, Sci. Rep. 3, 1781 (2013)

[34] F. F. Fanchini, G. Karpat, B. Çakmak, L. K. Castelano, G. H. Aguilar, O. J. Farías, S. P. Walborn, P. H. Souto Ribeiro, and M. C. de Oliveira, Phys. Rev. Lett. 112, 210402 (2014).

[35] A. Orieux, A. D’Arrigo, G. Ferranti, R. L. Franco, G. Benenti, E. Paladino, G. Falci, F. Sciarrino, and P. Mataloni, Sci. Rep. 5, 8575 (2015).

[36] J. Jin, V. Giovannetti, R. Fazio, F. Sciarrino, P. Mataloni, A. Crespi, and R. Osellame, Phys. Rev. A 91, 012122 (2015).

[37] S. Gröblacher, A. Trubarov, N. Prigge, G. D. Cole, M. Aspelmeyer, and J. Eisert, Nat. Commun. 6, 7606 (2015).

[38] N. K. Bernardes, A. Cuevas, A. Orieux, C. H. Monken, P. Mataloni, F. Sciarrino, and M. F. Santos, Sci. Rep. 5, 17520 (2015).

[39] N. K. Bernardes, J. P. S. Peterson, R. S. Sarthour, A. M. Souza, C. H. Monken, I. Roditi, I. S. Oliveira, and M. F. Santos, Sci. Rep. 6, 33945 (2016).

[40] W. F. Stinespring, Proc. Am. Math. Soc. 6, 211 (1955). 
[41] G. Adesso, S. Ragy, and A. R. Lee, Open Syst. Inf. Dyn. 21, 1440001 (2014).

[42] H. A. Haus and J. A. Mullen, Phys. Rev. 128, 2407 (1962).

[43] C. M. Caves, Phys. Rev. D 26, 1817 (1982).

[44] A. A. Clerk, M. H. Devoret, S. M. Girvin, F. Marquardt, and R. J. Schoelkopf, Rev. Mod. Phys. 82, 1155 (2010).

[45] E. Eleftheriadou, S. M. Barnett, and J. Jeffers, Phys. Rev. Lett. 111, 213601 (2013).

[46] G. Adesso and F. Illuminati, J. Phys. A 40, 7821 (2007).

[47] A. Ferraro, S. Olivares, and M. G. A. Paris, Gaussian States in Continuous Variable Quantum Information, Napoli Series on Physics and Astrophysics (Bibliopolis, Napoli, 2005).

[48] R. Simon, N. Mukunda, and B. Dutta, Phys. Rev. A 49, 1567 (1994).

[49] J. Eisert, S. Scheel, and M. B. Plenio, Phys. Rev. Lett. 89, 137903 (2002).

[50] J. Fiurášek, Phys. Rev. Lett. 89, 137904 (2002).

[51] G. Giedke and J. I. Cirac, Phys. Rev. A 66, 032316 (2002).

[52] G. Lindblad, J. Phys. A 33, 5059 (2000).

[53] T. M. Heinosaari, A. S. Holevo, and M. M. Wolf, Quantum Inf. Comput. 10, 619 (2010).

[54] G. De Palma, A. Mari, V. Giovannetti, and A. S. Holevo, J. Math. Phys. (N.Y.) 56, 052202 (2015).
[55] M.-D. Choi, Linear Algebra Appl. 10, 285 (1975).

[56] See Supplemental Material at http://link.aps.org/ supplemental/10.1103/PhysRevLett.118.050401 for technical proofs.

[57] A finite-dimensional channel acting on a $d$-dimensional system is $d^{\prime}$-positive if its extension to the system plus a $d^{\prime}$-dimensional ancilla is a positive map; then, said map is CP (i.e., $\infty$-positive) if and only if it is $d$-positive. Here, in the Gaussian scenario, we define instead $k$-positivity in terms of the extension to a number $k$ of ancillary CV modes. Since a single mode is already infinite dimensional, it is perhaps not surprising that complete positivity can be recovered already for $k=1$ in our framework.

[58] J. Schäfer, E. Karpov, R. García-Patrón, O. V. Pilyavets, and N. J. Cerf, Phys. Rev. Lett. 111, 030503 (2013).

[59] V. Giovannetti, R. García-Patròn, N. J. Cerf, and A. S. Holevo, Nat. Photonics 8, 796 (2014).

[60] H. P. Breuer and F. Petruccione, The Theory of Open Quantum Systems (Oxford University Press, New York, 2002).

[61] M. Schlosshauer, Decoherence and the Quantum-toClassical Transition, The Frontiers Collection No. 1 (Springer-Verlag, Berlin, 2007).

[62] G. Guarnieri, J. Nokkala, R. Schmidt, S. Maniscalco, and B. Vacchini, Phys. Rev. A 94, 062101 (2016). 